\title{
Heavy Neutron Stars? A Status Report on Arecibo Timing of Four Pulsar - White Dwarf Systems
}

\author{
David J. Nice, Eric M. Splaver \\ Physics Department, Princeton University \\ Box 708, Princeton, NJ 08544 USA
}

Ingrid H. Stairs

Department of Physics and Astronomy, University of British Columbia, 6224 Agricultural Road, Vancouver, BC V6T 1Z1, Canada

\begin{abstract}
Relativistic phenomena (orbital precession, Shapiro delay, and/or orbital decay) have been measured in Arecibo timing observations of four pulsar-white dwarf binaries, leading to constraints on the neutron star masses. We have detected the decay of the PSR J0751+1807 orbit due to gravitational radiation emission, the first such measurement in a binary with a low mass ratio $\left(m_{2} / m_{1} \ll 1\right)$. Timing data constrains the mass of this pulsar to bet between 1.6 and $2.8 \mathrm{M}_{\odot}$. Masses of the other pulsars are in marginal agreement with the canonical pulsar mass of $1.35 \mathrm{M}_{\odot}$, but higher values are preferred.
\end{abstract}

\section{Introduction}

The conventional wisdom is that all pulsars have mass close to the Chandrasekhar value, $\sim 1.35 \mathrm{M}_{\odot}$. This belief has arisen from high precision measurements of neutron star-neutron star binary systems; in all cases, the pulsar and the secondary neutron star have masses within a narrow range, 1.33 to $1.45 \mathrm{M}_{\odot}$ (Stairs et al. 2002; Weisberg \& Taylor 2003; Deich \& Kulkarni 1996). Perhaps all neutron stars are born within this narrow mass range, or perhaps the particular evolution of double neutron star leads to this result (Brown 1995). Even if all neutron stars are born near the Chandrasekhar mass, they may achieve higher masses through accretion of matter from orbital companions. Millisecond pulsars in orbits with white dwarfs, in particular, are known to have accreted mass during their low-mass X-ray binary phases, and hence they are prime candidates to be relatively massive pulsars. There has been some observational evidence for heavy pulsars in these systems (Kaspi, Taylor \& Ryba 1994; van Straten et al. 2001), although a statistical study argued that they are no heavier than pulsars in double neutron star binaries (Thorsett \& Chakrabarty 1999).

In this status report, we summarize observations of four millisecond pulsars in pulsar-white dwarf binaries made with the Arecibo telescope. This is an update of Nice, Splaver \& Stairs (2003), incorporating significant new timing data from PSR J0751+1807 and an improved timing model (including orbitalannual parallax) for PSR J1713+0747. 
Table 1. Mass measurements from timing analysis.

\begin{tabular}{lcccc}
\hline Pulsar & $\begin{array}{c}\text { Orbital } \\
\text { Period } \\
P_{b} \text { (days) }\end{array}$ & Eccentricity & $\begin{array}{c}\text { Mass } \\
\text { Function } \\
f_{1}\left(\mathrm{M}_{\odot}\right)\end{array}$ & $\begin{array}{c}\text { Pulsar Mass } \\
(95 \% \text { confidence) } \\
m_{1}\left(\mathrm{M}_{\odot}\right)\end{array}$ \\
\hline $\mathrm{J} 0621+1002$ & 8.32 & 0.002457 & 0.0270 & $1.1-2.3$ \\
$\mathrm{~J} 0751+1807$ & 0.26 & 0.000003 & 0.0010 & $1.6-2.8$ \\
$\mathrm{~J} 1713+0747$ & 67.83 & 0.000075 & 0.0079 & $1.2-2.1$ \\
$\mathrm{~B} 1855+09$ & 12.33 & 0.000022 & 0.0056 & $1.4-1.8$ \\
\hline
\end{tabular}

\section{Observations and Data Reduction}

Observations were made at Arecibo over several years at radio frequencies 430 and $1410 \mathrm{MHz}$, primarily using the Princeton Mark IV data acquisition system (Stairs et al. 2000). Details will be given elsewhere. These data were augmented by observations at Green Bank (140 Foot telescope), Jodrell Bank, and Effelsberg, as well as data collected at Arecibo using the Arecibo Berkeley Pulsar Processor and the Princeton Mark III system.

Pulse arrival times were derived using conventional methods and were fit to timing models using TEMPO. ${ }^{1}$ For each pulsar binary, at least one postKeplerian orbital parameter is detectable, leading to constraints on pulsar mass, $m_{1}$, secondary star mass, $m_{2}$, and orbital inclination, $i$ (see Taylor 1992 for the relations between post-Keplerian parameters, masses, and orbital inclination). To derive confidence intervals on $m_{1}, m_{2}$, and $i$, we calculated the goodness-offit of timing solutions over a grid of trial values of $\cos i$ and $m_{2}$. Each pair of trial values corresponds to a particular pulsar mass, $m_{1}$, according to the mass function, $f_{1}=\left(m_{2} \sin i\right)^{3}\left(m_{1}+m_{2}\right)^{-2}=\left(2 \pi / P_{b}\right)^{2} x^{3} / T_{\odot}$, where $T_{\odot}=4.925 \mu \mathrm{s}$ is the solar mass in time units, $P_{b}$ is the orbital period, $x$ is the semi-major axis projected into the line-of-sight in time units, and $m_{1}$ and $m_{2}$ are in solar mass units. For each trial, relativistic post-Keplerian parameters appropriate to $\cos i$ and $m_{2}$ were calculated and held constant, while all other parameters were fit.

The contours plotted in Figure 1 show the combinations of $\cos i$ and $m_{1}$ that gave acceptable fits for each source. We expect $\cos i$ to be uniformly distributed for randomly oriented binary systems. (Other than this, the intrinsic values of $i$ are of limited interest.) Table 1 summarizes the pulsar parameters and $95 \%$ confidence limits on pulsar masses from the timing data.

\section{Individual Sources}

PSR J0621+1002: This is an "intermediate mass" binary millisecond pulsar, presumably with a $\mathrm{CO}$ white dwarf secondary. Constraints on the masses come from the relativistic advance of periastron - we measure the precession rate to be $\dot{\omega}=0.0116 \pm 0.0008 \mathrm{yr}^{-1}(1 \sigma)$, yielding $m_{1}+m_{2}=2.8 \pm 0.3 \mathrm{M}_{\odot}-$ and

\footnotetext{
${ }^{1}$ See http://pulsar.princeton.edu/tempo
} 


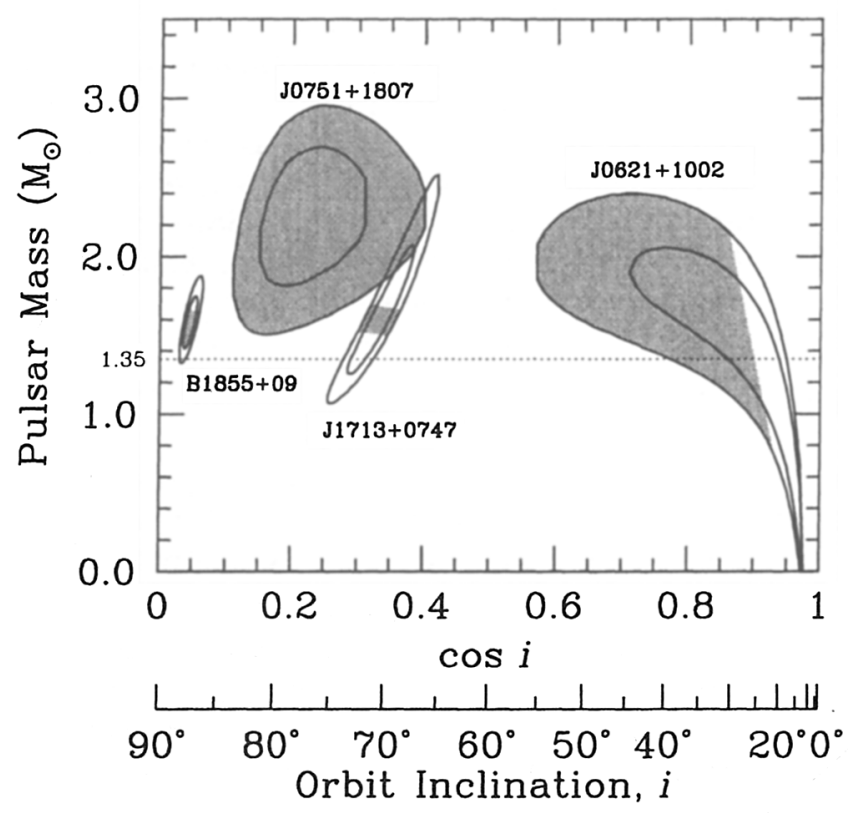

Figure 1. Constraints on pulsar masses and orbital inclinations. Inner and outer contours are $68 \%$ and $95 \%$ confidence regions, respectively, based on timing data alone. Shaded areas indicate theoretical limits within these contours, from the assumption that the companion is a white dwarf (J0621+1002) and from predictions of secondary star masses from the $P_{b}-m_{2}$ relation (B1855+09 and J1713+0747). (There are no theory constraints on J0751+1807.)

from the lack of detection of Shapiro delay, which excludes large inclinations. While the canonical pulsar mass value of $1.35 \mathrm{M}_{\odot}$ falls within the allowed region (Fig. 1), higher values are preferred, especially when we include the constraint that the white dwarf secondary have mass less than $1.4 \mathrm{M}_{\odot}$ (shading in Fig. 1). See Splaver et al. (2002) for more details.

PSR J0751+1807: This pulsar has a very short orbital period. By combining pre-upgrade (1993-1994) and post-upgrade (1999-2003) Arecibo observations of this source, along with data from Jodrell Bank and Effelsberg, we are able to detect the orbital decay due to emission of gravitational radiation. The rate of change of orbital period is $\dot{P}_{b}=(-6.2 \pm 1.1) \times 10^{-14}$. This is the first detection of the decay of a pulsar-white dwarf orbit with a low mass ratio $\left(m_{2} / m_{1} \ll 1\right) .^{2}$ Shapiro delay is marginally detected, constraining $i$ to intermediate values. If the Shapiro delay detection can be confirmed and refined, it will provide independent constraints on the system masses, allowing for novel tests

\footnotetext{
${ }^{2}$ Note also the spectacular results from PSR J1141-6545, a young pulsar in an eccentric orbit with a massive white dwarf (Bailes et al. 2003).
} 
of relativistic gravity (Arzoumanian 2003). In any case, the allowed values of $m_{1}$ are significantly greater than $1.35 M_{\odot}$ (Fig. 1).

PSRs B1855+09 \& J1713+0747: These pulsars are in wide orbits with light white dwarf companions. In both cases, the Shapiro delay is detectable, constraining $i$ and $m_{1}$ as shown in Figure 1 . Binary evolution theory posits a narrow range of companion masses, $m_{2}$, for a given orbital period, $P_{b}$, in these systems (Rappaport et al. 1995; Tauris \& Savonije 1999; Podsiadlowski, Rappaport \& Pfahl 2002). The resulting constraints on $m_{1}$ and $i$ are indicated in Figure 1. Once again, values of $m_{1}$ somewhat higher than $1.35 \mathrm{M}_{\odot}$ are preferred. ${ }^{3}$

\section{Conclusion}

The sources individually provide some evidence for pulsars substantially more massive than the Chandrasekhar value, and the collection of constraints shown in Figure 1 provides a compelling case for heavy neutron stars.

Acknowledgments. The Arecibo Observatory is operated by Cornell University under a cooperative agreement with the NSF. Pulsar research at Princeton University is supported by NSF grant 0206205 . I. H. S. is supported by an NSERC UFA and Discovery Grant. We thank numerous collaborators, particularly Jim Cordes, who provided crucial early data on PSR J0751+1807.

\section{References}

Arzoumanian, Z. 2003, in ASP Conf. Series, Vol. 302, Radio Pulsars, eds. M. Bailes et al., (San Francisco: ASP), p. 69

Bailes, M, Ord, S. M., Knight, H. S., \& Hotan, A W. 2003, ApJ, 595, 49

Brown, G. E. 1995, ApJ, 440, 270

Deich, W. T. S., \& Kulkarni, S. R. 1996, in IAU Symp. 165, Compact Stars in Binaries, eds. J. van Paradijs et al., (Dordrecht: Kluwer), p. 279

Kaspi, V. M., Taylor, J. H., \& Ryba, M. F. 1994, ApJ, 428, 713

Nice, D. J., Splaver, E. M., \& Stairs, I. H. 2003, in ASP Conf. Series, Vol. 302, Radio Pulsars, eds. M. Bailes et al., (San Francisco: ASP), p. 75

Podsiadlowski, P., Rappaport, S., \& Pfahl, E. D. 2002, ApJ, 565, 1107

Rappaport, S., et al. 1995, MNRAS, 273, 731

Splaver, E. M., et al. 2002, ApJ, 581, 509

Stairs, I. H., Thorsett, S. E., Taylor, J. H., \& Wolszczan, A. 2002, ApJ, 581, 501

Tauris, T. M., \& Savonije, G. J. 1999, A\&A, 350, 928

Taylor, J. H. 1992, Phil. Trans. R. Soc. Lond. A, 341, 117

Thorsett, S. E., \& Chakrabarty, D. 1999, ApJ, 512, 288

Weisberg, J. M., \& Taylor, J. H. 2003, in ASP Conf. Series, Vol. 302, Radio Pulsars, eds. M. Bailes et al., (San Francisco: ASP), p. 93

van Straten, W. et al. 2001, Nature, 412, 158

\footnotetext{
${ }^{3}$ Parkes pulsar J0437-4715 gives a similar result, $m_{1}=1.58 \pm 0.18 \mathrm{M}_{\odot}$ (van Straten et al. 2001).
} 